\title{
Nitrogen cost minimization is promoted by structural changes in the transcriptome of $\mathrm{N}$-deprived Prochlorococcus cells
}

\author{
Robert W Read ${ }^{1}$, Paul M Berube ${ }^{2}$, Steven J Biller ${ }^{2}$, Iva Neveux ${ }^{1}$, Andres Cubillos-Ruiz ${ }^{2,3}$, \\ Sallie W Chisholm ${ }^{2,4}$ and Joseph J Grzymski ${ }^{1}$ \\ ${ }^{1}$ Division of Earth and Ecosystem Sciences, Desert Research Institute, Reno, NV, USA; ${ }^{2}$ Department of Civil \\ and Environmental Engineering, Massachusetts Institute of Technology, Cambridge, MA, USA; ${ }^{3}$ Microbiology \\ Graduate Program, Massachusetts Institute of Technology, Cambridge, MA, USA and ${ }^{4}$ Department of Biology, \\ Massachusetts Institute of Technology, Cambridge, MA, USA
}

\begin{abstract}
Prochlorococcus is a globally abundant marine cyanobacterium with many adaptations that reduce cellular nutrient requirements, facilitating growth in its nutrient-poor environment. One such genomic adaptation is the preferential utilization of amino acids containing fewer $\mathrm{N}$-atoms, which minimizes cellular nitrogen requirements. We predicted that transcriptional regulation might further reduce cellular $\mathbf{N}$ budgets during transient $\mathbf{N}$ limitation. To explore this, we compared transcription start sites (TSSs) in Prochlorococcus MED4 under N-deprived and N-replete conditions. Of 64 genes with primary and internal TSSs in both conditions, $\mathrm{N}$-deprived cells initiated transcription downstream of primary TSSs more frequently than N-replete cells. Additionally, 117 genes with only an internal TSS demonstrated increased internal transcription under $\mathrm{N}$-deprivation. These shortened transcripts encode predicted proteins with an average of $21 \%$ less $\mathrm{N}$ content compared to full-length transcripts. We hypothesized that low translation rates, which afford greater control over protein abundances, would be beneficial to relatively slow-growing organisms like Prochlorococcus. Consistent with this idea, we found that Prochlorococcus exhibits greater usage of glycine-glycine motifs, which causes translational pausing, when compared to faster growing microbes. Our findings indicate that structural changes occur within the Prochlorococcus MED4 transcriptome during N-deprivation, potentially altering the size and structure of proteins expressed under nutrient limitation.
\end{abstract}

The ISME Journal (2017) 11, 2267-2278; doi:10.1038/ismej.2017.88; published online 6 June 2017

\section{Introduction}

Primary productivity is limited by nitrogen (N) availability in many ocean ecosystems (Tyrrell, 1999; Deutsch et al., 2007; Moore et al., 2013), and organisms that live there have adaptations that help them survive in low nutrient conditions. These adaptations include small cell sizes, which facilitate nutrient transport by increasing surface:volume ratios and reducing the absolute cellular requirement for nutrients (Munk and Riley, 1952; Gavis, 1976; Chisholm, 1992), as well as small genomes and a proteome that is $\mathrm{N}$ cost minimized (Grzymski and Dussaq, 2012). The concept of cost minimization was originally based on an observation that assimilatory proteins for a specific element contain relatively less of that specific element than average proteins in the

Correspondence: JJ Grzymski, Department of Earth and Ecosystem Sciences, Desert Research Institute, 2215 Raggio Parkway, Reno, NV 89509, USA.

E-mail: joeg@dri.edu

Received 2 November 2016; revised 20 April 2017; accepted 28 April 2017; published online 6 June 2017 same organism (Baudouin-Cornu et al., 2001). For many oligotrophic microorganisms, however, cost minimization extends beyond the proteins involved in assimilation and is observed across the proteome; for example, the genomes of $\mathrm{N}$ cost minimized oligotrophic microbes code for proteins that are, on average, reduced in amino acids containing added $\mathrm{N}$ side chains as compared to their coastal counterparts (Grzymski and Dussaq, 2012). As an evolutionary trade-off, the proteomes of N-cost- minimized organisms typically have slightly larger mass or more C atoms - an element which is not usually limiting for marine autotrophs (Grzymski and Dussaq, 2012).

The marine cyanobacterium Prochlorococcus MED4 is a striking example of a N-cost- minimized organism (Grzymski and Dussaq, 2012). Prochlorococcus is often the numerically dominant primary producer in oligotrophic waters (Flombaum et al., 2013), and can be broadly divided into two main subgroups, made up of high-light-adapted and lowlight-adapted cells. High-light-adapted Prochlorococcus cells are the smallest cyanobacterial cells in the oceans, and also have the smallest genomes of 
any free-living photosynthetic cell. The abundance of high-light-adapted Prochlorococcus cells typically exceeds that of low-light-adapted cells by several orders of magnitude in surface waters (Ahlgren et al., 2006; Zinser et al., 2006, 2007; Malmstrom et al., 2010). The divergence of high-light-adapted Prochlorococcus from their low-light-adapted relatives is correlated with a reduction in the overall $G$ $+\mathrm{C}$ content of their genomes, thereby reducing the $\mathrm{N}$ requirements of these cells due to the bias of low $\mathrm{G}$ $+\mathrm{C}$ codons to encode low $\mathrm{N}$-containing amino acids (Grzymski and Dussaq, 2012).

In addition to reducing the $\mathrm{N}$ content of their proteomes, high-light-adapted Prochlorococcus have undergone a process of genome streamlining in which number of coding sequences have been reduced, in part, by dispensing with most regulatory proteins (Grzymski and Dussaq, 2012; Giovannoni et al., 2014). How, then, do these cells respond to changing environmental conditions? Recent studies have revealed that, in addition to traditional protein regulators (transcription factors, sigma factors, etc.), many bacteria also utilize RNA-based mechanisms to regulate transcript abundance (Sharma et al., 2010). Prochlorococcus, like other streamlined microbes, such as Helicobacter pylori, has an unexpectedly complex transcriptome for a small genome, that includes cis and trans regulating RNA, other noncoding RNAs, varying cistronic and polycistronic operon regulation, and mRNAs with short half-lives (Steglich et al., 2010; Voigt et al., 2014). These regulatory characteristics likely enable responses to environmental signals without a complex proteinbased regulatory network.

Given the degree to which N cost minimization has been selected for in the genome of Prochlorococcus MED4 over evolutionary time, we wondered whether the transcriptional response in these cells has also been optimized to further reduce $\mathrm{N}$ requirements. We hypothesized that transcriptome changes could help provide additional $\mathrm{N}$ savings during nutrient limited growth-a concept we define as transcriptomic cost minimization. To test this hypothesis, we compared the primary transcriptomes (the set of unprocessed transcripts that allow experimental determination of transcription initiation sites) of Prochlorococcus MED4 grown under $\mathrm{N}$-deprived and N-replete conditions, allowing us to examine alterations in the transcriptional start sites under N-deprivation. Our results uncovered responses to $\mathrm{N}$ stress that highlight the capacity of already 'streamlined' cells to dynamically minimize their $\mathrm{N}$ requirements in response to $\mathrm{N}$-deprivation.

\section{Materials and methods}

\section{Cell cultures}

Axenic Prochlorococcus MED4 cells were grown in batch culture at $25^{\circ} \mathrm{C}$ under continuous illumination of $\sim 55 \mu \mathrm{mol}$ photons $\mathrm{m}^{-2} \mathrm{~s}^{-1}$ using cool, white, fluorescent bulbs. Cultures were grown in Pro99 media (Moore et al., 2007), prepared with $0.2 \mu \mathrm{m}$ filtered seawater collected from a depth of $20 \mathrm{~m}$ in the South Pacific Subtropical Gyre (26.25으, $104{ }^{\circ} \mathrm{W}$ ). Background inorganic nitrogen concentrations for this seawater, measured on-board, were typically below detection limits of $0.1,0.01$, and $0.02 \mu \mathrm{M}$ for nitrate+nitrite, nitrite, and ammonium, respectively. Of these nitrogen sources, Prochlorococcus MED4 can only assimilate ammonium, which was supplied in the amended medium at a concentration of $800 \mu \mathrm{M}$ (Moore et al., 2010). The medium was further amended with $3.75 \mathrm{~mm}$ TAPS buffer $(\mathrm{pH}=8)$ and $6 \mathrm{~mm}$ sodium bicarbonate to control $\mathrm{pH}$. Cells were allowed to acclimate to these temperature, light and media conditions by successively transferring the cultures four times during midexponential phase ( $\sim 20$ generations).

Triplicate $4.5 \mathrm{l}$ batch cultures were grown in $10 \mathrm{l}$ clear polycarbonate carboys. Bulk culture fluorescence was measured daily using a 10 AU fluorometer (Turner Designs, Sunnyvale, CA, USA). Cells were preserved for flow cytometry by fixation in $0.125 \%$ glutaraldehyde and storage at $-80^{\circ} \mathrm{C}$. Specific growth rates of replicates in the $4.5 \mathrm{l}$ cultures were estimated from the log-linear portion of growth curves constructed from bulk culture fluorescence and cell counts obtained from flow cytometry analysis. For these batch source cultures, fluorescence-based measurements yielded a growth rate of $0.60 \mathrm{~d}^{-1} \pm 0.01$ (1 standard deviation) and flow cytometry-based measurements yielded a growth rate of $0.67 \mathrm{~d}^{-1} \pm 0.14$ (1 standard deviation). Once the cultures reached mid-exponential phase, $3.8 \mathrm{l}$ of each culture was concentrated by centrifugation in four $1 \mathrm{l}$ centrifuge bottles (Beckman Coulter, Brea, CA, USA) using a JLA-8.1000 rotor in an Avanti J-20 XP centrifuge (Beckman Coulter) at $25^{\circ} \mathrm{C}$ and $9000 \mathrm{~g}$ (6857 rpm) for $15 \mathrm{~min}$. Each cell pellet was washed once by re-suspension in $375 \mathrm{ml}$ of $\mathrm{N}$-depleted media (lacking ammonium chloride addition) and concentrated again by centrifugation. Half of the washed cells were re-suspended in $1.8 \mathrm{l}$ of fresh N-replete Pro99 media (containing $800 \mu \mathrm{M}$ ammonium chloride), and the remaining half of the washed cells were resuspended in $1.8 \mathrm{l}$ of fresh N-depleted Pro99 media (lacking ammonium chloride addition). The only difference between the control and experimental treatments was the concentration of ammonium.

Samples for bulk culture fluorescence, cell enumeration by flow cytometry, fluorescence induction measurements, and RNA extraction were collected at $0,3,12$ and $24 \mathrm{~h}$ following the re-suspension of cells in either nitrogen-replete or nitrogen-depleted media. At each time point, bulk culture fluorescence was measured using a $10 \mathrm{AU}$ fluorometer (Turner Designs), and cells were preserved for flow cytometry by fixation in $0.125 \%$ glutaraldehyde and storage at $-80^{\circ} \mathrm{C}$. Following the experiment, fixed cells were enumerated using an Influx Cell Sorter (BD Biosciences, San Jose CA, USA), as previously described (Olson et al., 1985; Cavender-Bares et al., 1999). 
Photochemical conversion efficiency (Fv/Fm) was measured using a FIRe Fluorescence Induction and Relaxation System (Satlantic, Halifax, NS, Canada); cells were acclimated in the dark for 30 min to relax photosynthetic reaction centers before fluorescence induction curves were obtained using single turnover flash with blue light (450 nM with $30 \mathrm{~nm}$ bandwidth). Raw data were processed using fireworx (Dr Audrey B Barnett, Dalhousie University, Halifax, NS, Canada) in MATLAB.

At each time point, biomass for RNA extraction was obtained by centrifugation of $250 \mathrm{ml}$ of culture in $250 \mathrm{ml}$-centrifuge bottles (Beckman Coulter) using a JA-14 rotor in an Avanti J-20 XP centrifuge (Beckman Coulter) at $25^{\circ} \mathrm{C}$ and $9000 \mathrm{~g}$ (9666 rpm) for $15 \mathrm{~min}$. Cell pellets were re-suspended in $750 \mu \mathrm{l}$ of RNA denaturation solution (Ambion ToTALLY RNA Total RNA Isolation Kit, Life Technologies, Grand Island, NY, USA) by pipetting, frozen in liquid nitrogen and stored at $-80^{\circ} \mathrm{C}$ until processing, at which point total RNA was extracted using the Ambion ToTALLY RNA kit (Life Technologies), yielding $\sim 12 \mu \mathrm{g}$ of total RNA from each pellet.

\section{RNA sequencing overview}

Total RNA from each sample was divided and used in two sequencing workflows: gene expression analysis using strand specific sequencing of terminator exonuclease (TEX)-treated RNA (all time points) and primary transcriptome analysis using $5^{\prime}$ rapid amplification of cDNA ends (RACE) tagRNAseq (12 and $24 \mathrm{~h}$ time points). For gene expression analysis, it is necessary to account for transcripts within the total RNA pool that have undergone triphosphate to monophosphate conversion for ultimate degradation (Celesnik et al., 2007; Schoenberg, 2007). A caveat of doing timeseries analysis of gene expression using total RNA is that these signals are not removed and changes can be obfuscated, especially if half-lives of RNA are pathway or genedependent-a likely scenario with Prochlorococcus. The half-life differences of RNAs (Steglich et al., 2010) could play a significant role in interpreting differences in RNA treatment methodology. For these reasons, we chose to use TEX-treated RNA for gene expression analysis. For primary transcriptome analysis and determination of transcription start sites (TSSs), $5^{\prime}$ rapid amplification of cDNA ends tagRNA-seq was used because it selectively captures $5^{\prime}$ triphosphate ends at single nucleotide resolution. This produces the saw-tooth pattern, a dense clustering of nucleotides mapping to the $5^{\prime}$ end of the transcript, observed at transcriptional start sites throughout the genome (Sharma et al., 2010).

Ion Torrent sequencing for gene expression analysis RNA was treated with TEX (Epicentre, Madison, WI, USA) to remove rRNA contamination and sequenced at the Nevada Genomics Center (Reno, NV, USA).
Library preparation was performed using the Ion Total RNA-Seq Kit v2 (Life Technologies), which fragmented the total RNA and used reverse transcriptase to produce cDNA for strand-specific sequencing on the Ion platform. Strand-specific ion torrent sequencing (Life Technologies) yielded $130 \mathrm{bp}$ reads. Raw sequencing reads were uploaded to the sequencing read archive (NCBI accession: SRP078366, SRX1939126), and were inspected using FastQC (Andrews, 2010) to determine quality, ambiguous read percentage and relative amount of sequence reads. Ion-torrent RNA sequencing resulted in an average of $2.62 \times 10^{7} \pm 5.77 \times 10^{6}$ raw reads per cDNA library for the TEX-treated, N-deprived cells and $2.29 \times 10^{7} \pm 6.99 \times 10^{6}$ reads for the TEX-treated, $\mathrm{N}$-replete cells. The average quality score was Q24 (Supplementary Table S1).

Sequencing reads were utilized as the input for Rockhopper (McClure et al., 2013) for bacterial RNAsequencing analysis. Seed length was $15 \%$ of the read length, and any read with mismatches greater than $15 \%$ of the read was disregarded. This removed approximately $10 \%$ of the reads from each treatment. Differential expression was calculated against a negative binomial distribution between the experimental (N-deprived) cells and the control (N-replete cells) at each time point $(0,3,12,24 \mathrm{~h}$ post $\mathrm{N}$ removal) using the DESeq algorithm (Anders and Huber, 2010; Tjaden, 2015). Significantly differentially abundant transcripts were determined as those with an adjusted $P$-value $<0.05$ when comparing the two treatments. The adjusted $P$-value controls the false discovery rate using the Benjamini-Hochberg procedure (Benjamini and Hochberg, 1995; McClure et al., 2013). Pearson correlation coefficients to previous research (Tolonen et al., 2006) were larger for genes in the top $50 \%$ of mean expression level; these differences are likely due to low read depth mapping in non-regulated genes. Therefore, expression changes are only reported for those transcripts with normalized Rockhopper expression values in the top $50 \%$ of the data set.

\section{Illumina sequencing for primary transcriptome analysis} To determine the primary transcriptome of MED4, we analyzed transcriptional mappings at 12 and $24 \mathrm{~h}$ post N-deprivation relative to the N-replete controls. Total RNA was treated with Ribo-Zero rRNA Removal Kit for bacteria (Epicentre) to remove rRNA and any processed transcripts with $5^{\prime}$ monophosphate ends. The samples were next treated with $5^{\prime}$ RNA polyphosphatase (Epicentre) in order to convert the remaining 5' triphosphate structures into 5' monophosphate ends in preparation for adapter ligation. 5' Illumina TruSeq adapters were ligated to the monophosphate groups of the transcripts and cDNA was synthesized. The tagged 5' cDNA fragments were then specifically amplified with PCR and sequenced using $5^{\prime}$ rapid amplification of cDNA ends tagRNA-seq on a NextSeq 500 system with 
$75 \mathrm{bp}$ read lengths (vertis Biotechnologie AG). To visualize the sequencing reads (NCBI accession: SRP078366, SRX1939254) and transcriptional changes, reads were mapped to the Prochlorococcus MED4 genome using the segemehl short read aligner (Hoffmann et al., 2009, 2014). Resulting sam files containing mapped reads were converted into sorted and indexed bam files using samtools (Li et al., 2009). A single alignment run of Transcription Start Site Annotation Regime (TSSAR) software was performed to determine start site differences between the transcriptional start site-enriched samples and nonenriched samples in the N-replete and N-depleted samples (Amman et al., 2014). In keeping with the definitions considered by the TSSAR algorithm, we classified TSSs located on the opposite strand of an annotated gene as antisense; TSSs located within $250 \mathrm{nt}$ upstream of the gene's annotated TSS as primary start sites; and TSSs within the annotated gene as internal (Amman et al., 2014). Downstream TSSs are start sites that were located less than $30 \mathrm{nt}$ downstream of an annotated gene (Amman et al., 2014). Orphan TSSs were those that did not fall into any of these four categories, and we noted that some TSSs can represent a combination of multiple categories. Transcription from internal start sites in the sense orientation yield mRNAs that are called intraRNAs. Parameters for determining start site changes were a $P$-value of $1 \times 10^{-10}$, a noise threshold of 4 and a merge range of 5 . All TSSs identified were handcurated by uploading the sorted bam files into the Integrative Genomics Viewer, which placed the reads in the correct position and orientation in relation to the annotated Prochlorococcus MED4 genome (Robinson et al., 2011; Thorvaldsdóttir et al., 2013). Positions with a read count difference of less than 100 between the enriched and non-enriched samples were ignored. The TSSs identified by TSSAR after 24-h post $\mathrm{N}$-depletion were used to determine which TSSs were conserved between 12 and $24 \mathrm{~h}$ post $\mathrm{N}$-depletion. Sorted bam files were opened in $\mathrm{R}$ and read coverage figures were produced using the ggplot2 package (Wickham, 2009). If both a primary and internal TSS were present, the abundance of transcripts derived from an internal TSS was calculated by comparing the read mapping at the internal TSS to the read mapping at the primary TSS for both the N-depleted and N-replete samples. The magnitude of internal transcription in each treatment was then compared against each other. Only internal TSSs resulting in transcript abundance greater than $10 \%$ compared to their primary TSS were used. If no primary TSS was present, transcription from internal TSS was calculated by directly comparing read mapping in the $\mathrm{N}$-depleted sample to read mapping in the N-replete sample.

\section{Multiple sequence alignment and protein threading} For thirty genes, we compared the structure of isoforms corresponding to those derived from transcripts initiated from internal start sites with Protein Data Bank
(PDB) proteins using the protein structure predicting algorithm Phyre2 (Mezulis et al., 2015). Corresponding structure predictions were then overlaid on top of each other using separate colors to highlight differences between the two structures. Full-length proteins were also uploaded to NCBI and a domain search was completed using the conserved domain database. A caveat of this method is that no kinetics can be established, thus the functional efficiency of these predicted proteins remains unknown.

\section{Motif analysis}

Motif analysis of the Gly-Gly motifs and analysis of the 12mer motifs for pyrimidine and purine frequencies were carried out according to published methods (Grzymski and Marsh, 2014). Gly-Gly motifs were calculated from coding regions broken up by triplets to account for codon frequencies.

\section{Results and discussion}

Physiological responses to $\mathrm{N}$-deprivation

Our experimental design was based on previous work examining abrupt $\mathrm{N}$ deprivation in Prochlorococcus MED4 (Tolonen et al., 2006), facilitating comparisons between previous microarray gene expression results and our analysis of the primary transcriptome. Exponentially growing cells were subjected to acute $\mathrm{N}$ stress by resuspension in media with no added $\mathrm{N}$ (N-depleted). N-replete media was used as a control. Bulk culture fluorescence values began to diverge three hours after resuspension in N-depleted media; the fluorescence of N-replete cultures continued to increase while the fluorescence of N-depleted cultures decreased (Figure 1). Maximum PSII photochemical efficiency, Fv/Fm, was used as a measure of the cells' physiological response to $\mathrm{N}$ deprivation (Parkhill et al., 2001). Fv/Fm values for both N-replete and N-deprived cells declined during the first three hours, likely due to the shock of centrifugation and resuspension, but stabilized in the N-replete cells within $12 \mathrm{~h}$. By contrast, Fv/Fm continued to decline in the $\mathrm{N}$-deprived cells during the course of the experiment (Figure 1). Following $24 \mathrm{~h}$ in the N-depleted media, the $\mathrm{N}$-deprived cultures had lower bulk fluorescence, fewer cell counts and lower $\mathrm{Fv} / \mathrm{Fm}$ values compared to N-replete cultures. These results are consistent with previous work (Tolonen et al., 2006) and indicate a significant physiological response of Prochlorococcus MED4 to N-deprivation.

MED4 exhibits a canonical gene expression response to N-deprivation

Using the Ion Torrent reads derived from TEXtreated RNA, we first examined overall gene expression patterns by comparing the transcriptomes of the $\mathrm{N}$-deprived and N-replete cells at three different time points following induction of $\mathrm{N}$ stress. Overall, the 


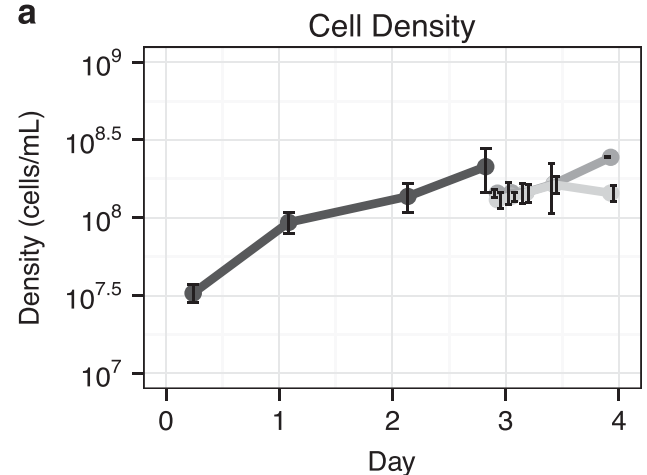

b

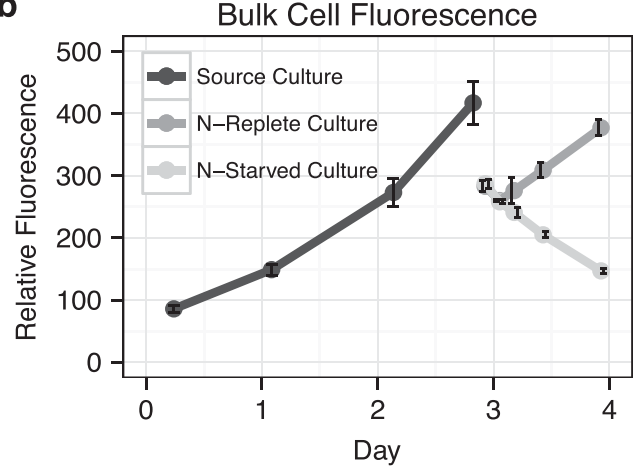

C

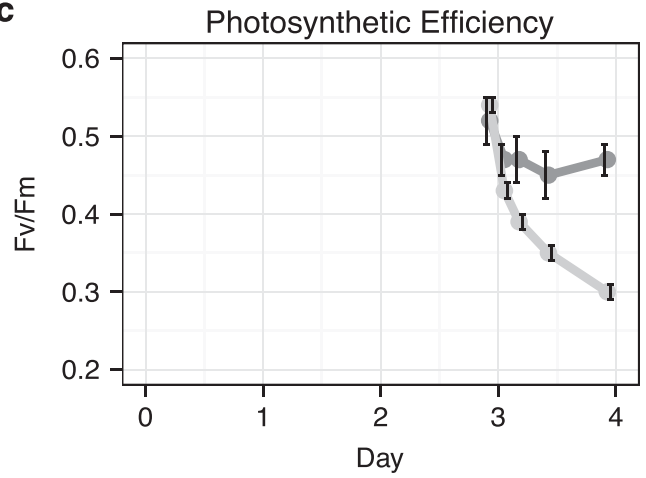

Figure 1 Physiological changes of Prochlorococcus MED4 during $\mathrm{N}$ deprivation. (a) Effect of $\mathrm{N}$ deprivation on cell concentration. (b) Effect of $\mathrm{N}$ deprivation on bulk culture fluorescence. (c) Effect of $\mathrm{N}$ deprivation on maximum quantum efficiency of photosystem II (Fv/ Fm) as measured by fast repetition rate fluorometry. Black lines represent the original triplicate cultures grown in N-replete media; other lines indicate cultures pelleted and resuspended in either N-replete (dark grey line) or N-deficient (light grey line) media. The discontinuity in fluorescence and cell concentration measurements result from incomplete recovery of cells following centrifugation. Error bars represent the standard deviation of three biological replicates and are smaller than the symbols when not visible.

gene expression changes we observed in the primary transcriptome in response to $\mathrm{N}$ deprivation were similar to changes previously observed in microarray analysis of total RNA (Tolonen et al., 2006) (Supplementary Text and Supplementary Table S2). We found that the relative changes of differentially expressed transcripts, between $\mathrm{N}$-deprived and N-replete cells, were approximately symmetrical; the number of transcripts demonstrating an increased abundance was similar to the number of transcripts
Table 1 Differentially expressed transcripts of $\mathrm{N}$-deprived vs N-replete cells $(P<0.05)$

\begin{tabular}{cccr}
\hline Time (h) Regulation & $\begin{array}{c}\text { Amount of differentially } \\
\text { expressed genes }\end{array}$ & $\begin{array}{c}\text { Differentially } \\
\text { expressed }\end{array}$ \\
\hline \multirow{3}{*}{3} & $\mathrm{Up}$ & 62 & $7.21 \%$ \\
12 & $\mathrm{Up}$ & 84 & $9.77 \%$ \\
24 & $\mathrm{Up}$ & 93 & $10.81 \%$ \\
3 & Down & 28 & $3.26 \%$ \\
12 & Down & 86 & $10.00 \%$ \\
24 & Down & 107 & $12.44 \%$ \\
\hline
\end{tabular}

exhibiting a decreased abundance. These data indicate a balanced expression response to $\mathrm{N}$ deprivation rather than decreasing transcription overall (Table 1). Down-regulation of a substantial fraction of genes would be a rapid N-saving mechanism and was not the case in Prochlorococcus MED4, indicating an active response to $\mathrm{N}$ deprivation compared to entry into stasis. Further, the transcriptional response was rapid, with significant changes in transcript abundance apparent within $3 \mathrm{~h}$ of $\mathrm{N}$-deprivation and additional changes noted after 12 and 24 h (Supplementary Tables S3-S6).

Cyanobacteria 'sense' N-deprivation by monitoring the C:N balance of the cell and respond to changes in this ratio by activating alternative $\mathrm{N}$ assimilation pathways when excess intracellular 2-oxoglutarate accumulates (Ohashi et al., 2011). The global nitrogen stress response regulator, NtcA (Sauer et al., 1999), is responsible for inducing the expression of assimilation pathways for a variety of $\mathrm{N}$ compounds. Consistent with previous reports (Tolonen et al., 2006), we found that members of the NtcA regulon are induced in response to $\mathrm{N}$ deprivation in Prochlorococcus MED4 (Supplementary Table S5). Members of the NtcA regulon that exhibited increased transcript abundance during $\mathrm{N}$-deprivation included genes encoding the assimilation pathways for urea and cyanate, as well as the glutamine synthase gene $\operatorname{gln} A$ (Supplementary Tables S3-S6). The NtcA regulon also regulates the urease enzyme and urea transporter enzyme. All subunits of the urease enzyme (ureA-C) were co-expressed and showed similar increases in relative abundance at $12 \mathrm{~h}$ post $\mathrm{N}$ deprivation. The urea transporter genes (urtA-E) were transcribed in a similar manner with the reads gathering at the TSS for urtA, the first gene in the operon (Supplementary Figure S1). In addition, 15 high light inducible proteins, known to respond to stress conditions in Prochlorococcus (Havaux et al., 2003; Tolonen et al., 2006; Steglich et al., 2008), increased in abundance after $12 \mathrm{~h}$ of $\mathrm{N}$ deprivation (Supplementary Table S7). One of these high light inducible proteins, Hli10, is encoded by a gene predicted to be regulated by NtcA (Tolonen et al., 2006).

Complexity of transcription initiation at variable start sites under $\mathrm{N}$-deprivation

Based on the Ion Torrent data set, gene expression changes in these Prochlorococcus MED4 cells 
exhibited responses similar to previous studies under N-deprivation (Tolonen et al., 2006; Gilbert and Fagan, 2011) suggesting that $\mathrm{N}$ savings cannot be achieved by simply down regulating transcription. Thus, to investigate whether Prochlorococcus might use other RNA-based regulatory mechanisms to reduce cellular $\mathrm{N}$ requirements under $\mathrm{N}$ stress, we next examined the primary transcriptome of MED4 following 12 and $24 \mathrm{~h}$ of $\mathrm{N}$ depletion. We performed detailed annotation of TSSs after $12 \mathrm{~h}$ post N-depletion due to the strong correlation of transcript patterns at this time point as compared to Tolonen et al. (2006), and confirmed these results with data from $24 \mathrm{~h}$ post N-depletion (Table 2, Supplementary Tables S8-S11).

Our detailed annotation of TSSs at 12 and $24 \mathrm{~h}$ following $\mathrm{N}$-depletion identified new internal and antisense TSSs (Table 2, Supplementary Tables S8S11) throughout the genome that were not previously reported by Voigt et al. (2014) for MED4 grown under N-replete conditions (Supplementary Text,Supplementary Table S12). For the purpose of this study, internal transcription is defined as a verified sawtooth pattern of transcript reads mapping downstream of a gene's annotated start site. Verification of the saw-tooth pattern was performed manually after running TSSAR. The internal transcription ratio is defined as the ratio of read abundance at the internal TSS compared to the abundance of reads at the primary TSS. The internal transcription ratio generally increased under N-deprivation (Figure 2; Supplementary Table S13), suggesting a specific regulatory response to $\mathrm{N}$ stress rather than erroneous transcription given that we did not observe degraded and random read mapping to these genes. Eighty-five genes contained both a primary TSS and internal TSS identified by TSSAR in both the N-depleted and N-replete samples (Supplementary Table S13). Based on a Wilcoxon rank sum test, 64 genes $(\sim 74 \%)$ demonstrated a significant increase in the internal transcription ratio $\left(P\right.$-value $<1 \times 10^{-6}$ for all 64 genes) in $\mathrm{N}$-depleted samples compared to the N-replete samples, with 13 genes containing multiple internal TSSs (for a total of 81 TSSs in these genes). After $24 \mathrm{~h}$ of $\mathrm{N}$ deprivation, transcripts expressed from $72 / 81(89 \%)$ of these internal TSSs (from 56 genes) were still present, indicating a conserved and continued role in the $\mathrm{N}$ stress response.

Transcription from an internal start site, however, was not exclusively a response to nutrient deprivation. We found the internal transcription ratio increased in 13 of the 85 genes (15\%) during $\mathrm{N}$-replete conditions compared to $\mathrm{N}$-deprivation, with an additional eight genes (11\%) exhibiting a similar internal transcription ratio in both the $\mathrm{N}$-replete and $\mathrm{N}$-depleted samples (Supplementary Table S13). Irrespective of condition (N-deplete vs $\mathrm{N}$-replete) 35 genes $(\sim 41 \%)$ showed a higher abundance of internal TSS reads compared to primary TSS reads, indicating a general increase in internal transcription for those genes. Combined with 187 TSSs from 160 genes that contain only an internal TSS, of which 117 (63\%) demonstrated greater internal transcription in $\mathrm{N}$-depleted cells, these results could indicate that some internal TSSs are actually the primary transcription start site which may have been incorrectly called. Consistent with this, Sharma et al. found 18 new gene start sites in their re-annotation of Helicobacter pylori using a similar method (Sharma et al., 2010).

On the other hand, we also observed that these sites are often located far downstream of the annotated start site and well within the expected coding sequence. This suggests that some of these internal TSSs may yield short sense RNAs (intraRNAs)-molecules which have been identified in other studies and are hypothesized to function as truncated alternative mRNAs or in other regulatory roles (Mitschke et al., 2011; Shao et al., 2014). Antisense TSSs were abundant in both N-replete and $\mathrm{N}$-deprived cells, however the relative number of them found throughout the genome was approximately equal in both treatments and cannot be correlated to gene expression changes. These findings further highlight the incredible complexity of transcriptional regulation in Prochlorococcus.

Transcriptional regulation and the $\mathrm{N}$-cost minimization of proteins

Many of the most abundant proteins found within microbes in the Sargasso Sea are transport proteins (Sowell et al., 2008), emphasizing their importance to bacteria living in extremely nutrient depleted environments. NtcA regulation of transporters is known to be an important part of the response of Prochlorococcus cells to N-deprivation, as evidenced by a marked increase in transcripts encoding the urea transporter Urt and ammonium transporter Amt1 under these conditions (Tolonen et al., 2006). Based on this, we next focused on the regulation of other genes with possible NtcA binding sites, which could allow them to be regulated as part of the NtcA regulon.

Table 2 Transcriptional start sites by category

\begin{tabular}{lccccccc}
\hline Type & $\begin{array}{c}\text { Downstream } \\
\text { antisense }\end{array}$ & $\begin{array}{c}\text { Internal } \\
\text { antisense }\end{array}$ & Internal & $\begin{array}{c}\text { Internal antisense } \\
\text { downstream }\end{array}$ & $\begin{array}{c}\text { Internal } \\
\text { primary }\end{array}$ & $\begin{array}{c}\text { Orphan Primary } \\
\text { Primary } \\
\text { antisense }\end{array}$ \\
\hline N-Replete & 6 & 363 & 502 & 1 & 122 & 124 & 542 \\
N-Starved & 8 & 275 & 519 & 5 & 116 & 118 & 477 \\
\hline
\end{tabular}



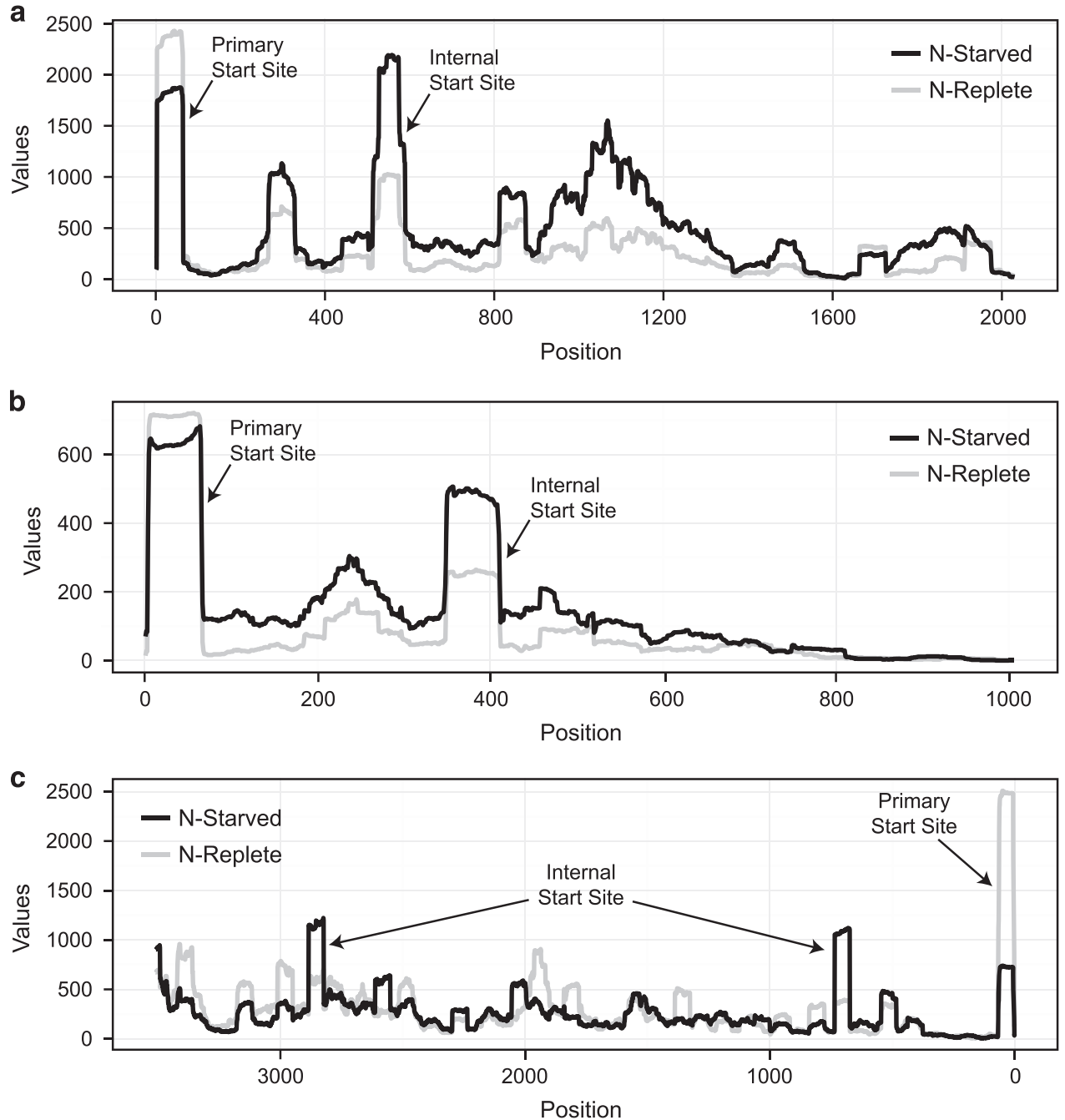

Figure 2 Primary transcriptome mapping to three genes during $\mathrm{N}$ deprivation. Values indicate the number of primary reads mapping to the (a). PMM0149 (ndhF), (b). PMM0058 (conserved hypothetical protein) and (c). PMM1485 (rpoB) genes. Experimental (black) and control reads (grey) were compared at $12 \mathrm{~h}$ post $\mathrm{N}$ deprivation. The primary and internal TSSs are marked with arrows in each panel. Each panel represents the full length of the gene, with the $x$-axis representing the distance from the primary start site.

As described above, N-deprivation increased internal transcription in Prochlorococcus MED4 (Figure 2). The intraRNAs initiated from these internal TSSs could have three possible functions: (1) they could be mis-transcribed, degraded or processed RNA with unknown or no function; (2) they could be structural RNAs or encode peptide scaffolds that are used for other regulatory purposes (Lybecker et al., 2014; Shao et al., 2014); or (3) they could be internally transcribed genes that code for functional proteins. While the intraRNAs could arise from transcriptional noise due to spurious promoterlike sequences, and in that case would be nonfunctional copies of RNA (Lybecker et al., 2014; Shao et al., 2014; Thomason et al., 2015), 245 genes with internal TSSs have clear regulatory differences in response to $\mathrm{N}$-deprivation (Figure 2). This has been seen in other cyanobacteria; for example, transcriptional mapping in Synechocystis sp. PCC6803 identified abundant short sense transcripts from internal TSSs, which were proposed to yield shorter isoforms of Synechocystis 6803 proteins (Mitschke et al., 2011). Random processes would not be expected to show reproducible responses to $\mathrm{N}$-deprivation (that is, an indication of transcription level regulation). Thus, it is likely these transcripts have a specific physiological role.

We observed genes with internal transcription under $\mathrm{N}$-deprived conditions were responsible for important physiological functions, such as RNA synthesis, glutamate synthesis, fatty acid biosynthesis, and transport of important ions such as sodium and cyanate. This fact led us to hypothesize that these intraRNAs may encode functional proteins, as the shortened RNA transcripts and shortened translated proteins would reduce the overall $\mathrm{N}$ requirements of the cell. Given that these cells were already nutrient stressed and exhibiting significantly reduced photosynthesis and growth rates (Figure 1), the cell might derive a net fitness benefit 

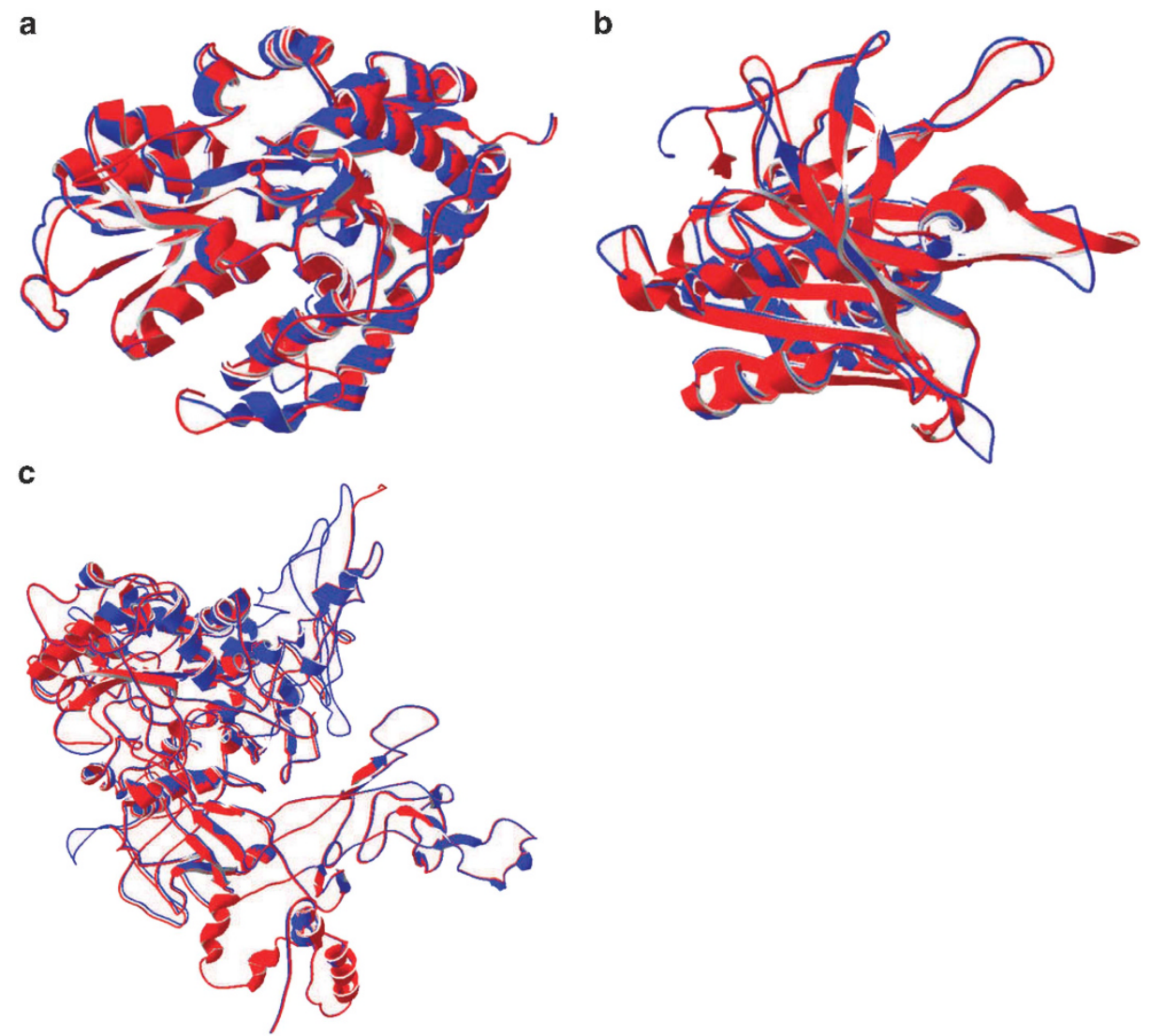

Figure 3 Structure predictions for internal transcription sites versus PDB structures. (a) Protein structure threading for the cyanate ABC transporter (PDB. c2i4cA). There are no major structural differences in the protein threads. Blue and red are the overlapping structure representing the full length transcript (blue) and the corresponding predicted protein from the internal start site in MED4 (red). (b) As in A, but for the Ferredoxin-NADP reductase protein (PDB C1jb9A). (c) Protein structure threading for RNA polymerase (PDB C3lu0C).

by expressing a partial protein which required fewer nutrients, as long as that protein retained at least some functionality. Of the 200 genes, which accounted for almost $90 \%$ of the total counts in the sequencing libraries, transcripts for 30 demonstrated evidence of internal start site usage (Supplementary Table S14). There were 18 total transcripts which aligned to all the structural elements of their counterpart PDB structure. These 18 shortened proteins would, if translated at equal levels, represent a savings to the cell of $2256 \mathrm{~mol} \mathrm{~N} \mathrm{~mol}^{-1}$ protein $(\sim 21 \%)$ compared to the full-length versions of these 18 proteins (range 10-41\%; Supplementary Table S14). Additional savings could be achieved at the RNA level as well.

To examine the functional impact of expressing shortened proteins, we evaluated the potential effect of the N-terminal truncation on the predicted functional domains of highly expressed genes using NCBI's conserved domain search (Supplementary Table S14). We also used multiple sequence alignments (MSA) and protein threading to predict the structure of proteins translated from intraRNAs by comparison to known structures (Supplementary Table S14). We predict that initiating translation at internal start sites would likely have one of three outcomes: (1) expression of proteins that align by multiple sequence alignments to all of the major structural elements of known structures and retain the major carbon backbone; (2) expression of proteins that lack all structural elements, or whose structure is clearly incomplete, or whose sequence was improperly annotated and; (3) expression of proteins where a few specific structural elements or domains are transcribed (Supplementary Table S14). The majority of the proteins examined, 22 of 30 , fall into categories 1 or 3 and are expected to be translated into functional proteins or domains (Supplementary Table S14).

Considering transcripts most relevant to N-stress, one of these-the cyanate ABC transporter (PMM0370)—evidences no structural differences between the full length translated protein and the shortened protein (Figure 3a, Supplementary Table S14). Although the full diversity of functional cyanate transport proteins with established PDB structures have yet to be discovered, our conserved domain analysis results suggest that the shortened protein retains full function of its two predicted domains (Supplementary Table S14). Still, the combined primary transcriptome and modeling results highlight how little we know about many bacterial proteins and further biochemical analysis are warranted to explore how changes in the primary amino acid sequence impact protein function. 
Additionally, two other non-transport proteins may have been expressed in shorter forms. For ferredoxin-NADP reductase, there are no discernible changes in the threaded protein structure of the internal transcript, and it likely retains full function of its single predicted domain (Figure 3b, Supplementary Table S14). RNA polymerase, vital for producing actual transcripts, has an internal TSS whose transcript encodes a protein that aligns to 95\% of the sequence from the known PDB structure from $E$. coli, although domain function could be slightly modified as the entire protein is predicted as a single domain (Figure 3c, Supplementary Table S14). It makes sense, physiologically, that under $\mathrm{N}$-stress conditions the protein responsible for producing transcripts is potentially $\mathrm{N}$ costminimized. If these three proteins were internally translated they would provide $541 \mathrm{~mol} \mathrm{~N} \mathrm{~mol}^{-1}$ translated protein savings relative to the fully translated proteins-approximately $17 \%$ savings.

In the context of the cell as a whole, transcriptomic $\mathrm{N}$ cost minimization is but one of several mechanisms through which Prochlorococcus cells adjust their elemental requirements in response to particular stressors, such as limitation for key elements such as iron, phosphorus, and nitrogen. For example, in response to iron limitation Prochlorococcus expresses the non-iron containing oxidoreductase flavodoxin instead of the iron-sulfur containing ferredoxin (Bibby et al., 2003; Thompson et al., 2011). Prochlorococcus also utilizes sulfolipids instead of phospholipids in its cellular membrane in order to decrease phosphorus requirements (Van Mooy et al., 2006). These responses are all based on the utilization of different pathways in order to modify the cell's elemental requirements. In contrast, transcriptomic cost minimization represents a response to nutrient limitation that depends on structural changes to the mRNA pool and, putatively, the proteome. While genomic $\mathrm{N}$ cost minimization, mediated by codon usage and general \% GC characteristics, can only change over evolutionary time scales, transcriptomic $\mathrm{N}$ cost minimization is a dynamic process which enables the cell to respond to changes in $\mathrm{N}$ availability on the order of hours.

Cost minimized organisms have a higher potential for decreased translation rates

An important consideration regarding both genomic and transcriptomic $\mathrm{N}$ cost minimization is that such $\mathrm{N}$ savings might only have a small overall impact if protein abundance is not tightly controlled. One strategy for maintaining careful control of protein levels is rapid RNA turnover (Steglich et al., 2010), which allows the cell to quickly adjust mRNA availability in response to stress. Prochlorococcus, for example, has a median RNA half-life on the order of 2 min, which is twofold faster than that observed in other microorganisms (Steglich et al., 2010). Cells can also improve their control of protein abundance by slowing down translation rates (Sherman and Qian, 2013). While faster growing (r-selected) organisms should experience selective pressures to maximize translation rates in order to support episodes of rapid growth, we hypothesize that slowly growing (k-selected), cost minimized organisms should instead experience selective pressures to minimize translation rates. To explore this hypothesis, we examined the genomes of both oligotrophic k-strategists and copiotrophic r-strategists to look for sequence signatures associated with translation rates.

Shine-Dalgarno motifs (ribosomal binding sites) internal to coding regions have been shown to cause translational pausing and subsequent reductions in growth rate (Li et al., 2012). These signatures are apparent in the observed-to-expected ratio of the frequency of the glycine-glycine motifs in coding regions. Depending on codon usage, Gly-Gly motifs can have high or low affinities to the anti-ShineDalgarno sequence found at the $3^{\prime}$ terminus of the $16 \mathrm{~S}$ rRNA in the ribosome to mediate pausing; such sequences are minimized in fast-growing organisms like E. coli (Li et al., 2012). We examined the occurrence of Gly-Gly motifs in the genomes of three bacteria with relatively high maximum growth rates (Bacillus cereus, E. coli K12 and Vibrio fisheri) and four oligotrophic, k-selected marine bacteria with relatively slow maximum growth rates (Prochlorococcus MED4, Prochlorococcus MIT9313, Synechococcus and Pelagibacter; Figure 4). We found that the genomes of the r-selected organisms exhibited a clear pattern of minimization of Gly-Gly motifs with high affinity to the anti-Shine-Dalgarno sequence, consistent with less pausing and faster protein expression. In oligotrophic k-selected organisms, the pattern is opposite with no apparent deviations in the observed-expected ratios of the motifs; in fact, the motifs with the highest binding affinity to the anti-Shine-Dalgarno sequence were found more often than expected (observed: expected $=1.63$ ) in Prochlorococcus MED4 than in oligotrophs (Fisher's exact test of normalized count $P$-values $<0.01)$. This suggests that selection against motifs that cause translational pausing is weak in oligotrophic k-selected organisms, and therefore that they would be expected to have slower translation rates than the r-strategists. This genomic feature represents yet another property that may contribute to cost minimization in oligotrophic microbes.

We propose that there are at least two mechanisms contributing to this weak selection against pausing sequences in slow-growing, oligotrophic bacteria. First, as previously discussed, translational pausing would be beneficial to k-selected organisms by improving the ability of cells to control protein abundance while growing in nutrient limited conditions. Second, it is likely that organisms such as Prochlorococcus do not rely on Shine-Dalgarno sequences for translation initiation (Voigt et al., 


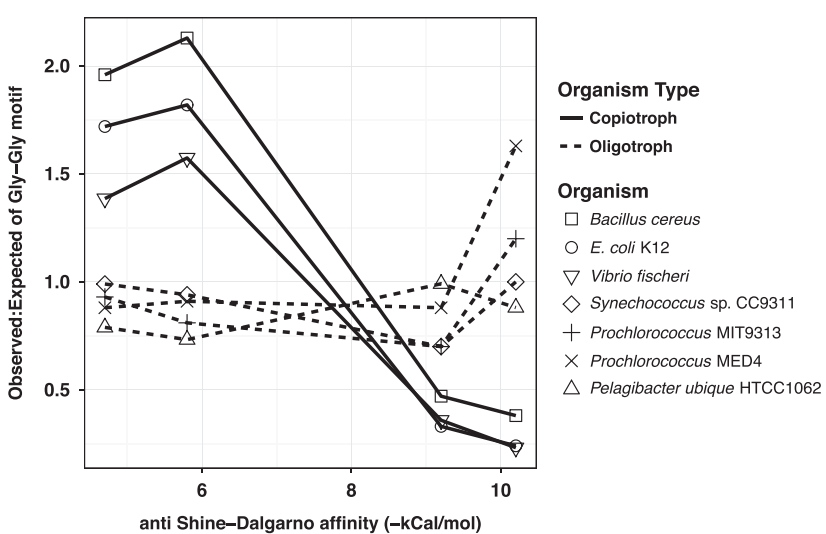

Figure 4 Frequency of Glycine-Glycine motifs in selected microbial genomes. Deviations in observed to expected ratios of Gly-Gly motifs, indicative of the potential for ribosomal stalling, are indicated for a set of common copiotrophic r-selected and oligotrophic k-selected organisms. The observed to expected ratio of gly-gly motifs are illustrated on the $y$-axis with the anti-ShineDalgarno affinity on the $x$-axis. Copiotrophs are represented by a solid line and oligotrophs a dashed line. Individual organisms are represented by the indicated shapes along the line.

2014). Instead, based on studies in Synechococcus, translation initiation may instead rely on an alternative mechanisms dependent on ribosomal protein S1 (Mutsuda and Sugiura, 2006; Voigt et al., 2014) or the direct binding of a $70 \mathrm{~S}$ monosome to leaderless mRNA start sites (Moll et al., 2002). This would reduce selection for canonical ribosomal binding sites and, as a less specific mechanism for translation initiation, might provide cells with a way to translate truncated proteins from mRNAs expressed from internal start sites under $\mathrm{N}$ stress. These k-selected organisms utilize less regulated and less specific transcription and translation mechanism. As many of these truncated transcripts are not predicted to contain canonical translation initiation sites, S1 binding could offer a mechanistic explanation for the translation of such proteins arising from unexpected transcriptional start sites.

To examine whether ribosomal protein S1-based translation initiation is likely to occur in Prochlorococcus MED4, we quantified the relative frequency of 10 and 12mer pyrimidine rich motifs (made up of at least $80 \%$ pyrimidines)—sequences which are conductive to S1 binding (Mutsuda and Sugiura, 2006). In connection with the pyrimidine rich regions, we also searched for NtcA binding regions based on the predicted motifs described previously (Su, 2005; Tolonen et al., 2006). Sites were counted as possible NtcA binding sites if they were found less than $100 \mathrm{bp}$ upstream of identified translational start sites, although in some published cases sites can be found much further upstream $(\mathrm{Su}, 2005)$. We found abundant 10 and 12 mer pyrimidine rich regions directly upstream of annotated translational start sites (Supplementary Table S15). Furthermore, we discovered that $287(\sim 17 \%)$ genes had possible NtcA binding sites, which could possibly facilitate NtcA regulation under $\mathrm{N}$ stress (Supplementary Table
S15). There are also 912 pyrimidine rich motifs found in non-coding regions of the genome (observed:expected $=1.35$, Fisher's exact test of normalized counts $P$-value $<0.05)$. Purine rich sequences are concomitantly under-observed and, given that there are no $\mathrm{G}+\mathrm{C}$ biases in purine and pyrimidine motifs, these observations are consistent with the hypothesis that S1 translation initiation can occur both in 5' untranslated regions and upstream of traditional start sites.

These data suggest that protein S1 could associate with these pyrimidine rich sequences and mediate translation of proteins expressed from the annotated primary start site or, as suggested by our primary transcriptome data under $\mathrm{N}$ deprivation conditions from internally transcribed start sites (Supplementary Table S14). We assume that translation initiates at methionine and our modeling results begin at the nearest downstream methionine to the TSS (Mutsuda and Sugiura, 2006). Leaderless mRNAs are likely present within Prochlorococcus MED4, requiring an alternate method of translation initiation. In these cases, mRNAs lacking a 5' UTR directly bind $70 \mathrm{~S}$ monosomes, thus initiating translation (Moll et al., 2002; Voigt et al., 2014). Our data, consistent with previous studies on Prochlorococcus MED4 (Voigt et al., 2014), suggest that $6-8 \%$ of all primary TSSs are found within 10nt of translation initiation sites and thus potentially initiated by $70 \mathrm{~S}$ monosomes.

\section{Conclusions}

In summary, we have shown that a variety of structural changes occur within the Prochlorococcus transcriptome in response to $\mathrm{N}$ deprivation, and that these changes likely contribute to the ability of this organism to minimize the amount of $\mathrm{N}$ required in its proteome. Specifically, Prochlorococcus increased the internal transcription ratio for 64 genes with both a primary and internal TSS and 117 genes with only an internal TSS during N stress conditions, which should in some cases produce shortened versions of enzymes that likely retain at least partial functionality and require fewer $\mathrm{N}$ atoms. Proteomic confirmation of the translation of the shortened peptides, as well as biochemical characterization, will be necessary to understand their abundance and function relative to their full-length versions. We also propose that Prochlorococcus may have relatively slow translation rates which, in conjunction with short RNA half-lives, allows them to control protein abundances, reducing cellular nitrogen requirements. These results provide an initial window onto the potential role for transcriptomic cost minimization during acute $\mathrm{N}$ starvation in Prochlorococcus, and in the future it will be interesting to determine whether this response contributes to other Prochlorococcus stress responses. Furthermore, to highlight the relevance of this study, future research should determine the degree of transcriptomic cost 
minimization in cells growing under chronic N-limitation, which would be more representative of the physiological status of Prochlorococcus cells in the oligotrophic ocean. Although the concept of cost minimization has heretofore been considered to function on evolutionary time scales (through selection on genomic codon usage to reduce nutrient requirements), these results show that cost minimization can encompass physiological mechanisms as well-through dynamic structural changes to the transcriptome that should result in a proteome requiring fewer $\mathrm{N}$ atoms. One hypothesis is that this mechanism functions as a resource reallocation strategy versus an active decrease of the overall $\mathrm{N}$ quota for the cell. Future studies should focus on producing more detailed quantitative analysis of the proteome using mass spectrometry and functional characterization of the truncated proteins that result from internal transcription.

\section{Conflict of Interest}

The authors declare no conflict of interest.

\section{Acknowledgements}

We thank Alexis Yelton (MIT) and Julie Miller (MIT) for assistance with sampling. We also thank Dr David Vuono (DRI) for his contributions in the preparation of the manuscript. This work was funded in part by the Gordon and Betty Moore Foundation through Grant GBMF495 to SWC, by a grant from the Simons Foundation (SCOPE award ID 329108 to SWC), and by the National Science Foundation (MCB-1244630 to JJG and OCE-1153588 and DBI-0424599 to SWC). ACR was supported by a HHMI International Student Research Fellowship. This article is a contribution from the Simons Collaboration on Ocean Processes and Ecology (SCOPE).

\section{References}

Ahlgren NA, Rocap G, Chisholm SW. (2006). Measurement of Prochlorococcus ecotypes using real-time polymerase chain reaction reveals different abundances of genotypes with similar light physiologies. Environ Microbiol 8: 441-454.

Amman F, Wolfinger MT, Lorenz R, Hofacker IL, Stadler PF, Findeiß S. (2014). TSSAR: TSS annotation regime for dRNA-seq data. BMC Bioinformatics 15: 89.

Anders S, Huber W. (2010). Differential expression analysis for sequence count data. Genome Biol 11: R106.

Andrews S. (2010). FastQC: a quality control tool for high throughput sequence data. Available online at: http:// www.bioinformatics.babraham.ac.uk/projects/fastqc.

Baudouin-Cornu P, Surdin-Kerjan Y, Marlière P, Thomas D. (2001). Molecular evolution of protein atomic composition. Science 293: 297-300.

Benjamini Y, Hochberg Y. (1995). Controlling the False Discovery Rate: A Practical and Powerful Approach to Multiple Testing. J R Stat Soc B 57: 289-300.

Bibby TS, Mary I, Nield J, Partensky F, Barber J. (2003). Low-light-adapted Prochlorococcus species possess specific antennae for each photosystem. Nature 424: 1051-1054.

Cavender-Bares KK, Mann EL, Chisholm SW, Ondrusek ME, Bidigare RR. (1999). Differential response of equatorial Pacific phytoplankton to iron fertilization. Limnol Oceanogr 44: 237-246.

Celesnik H, Deana A, Belasco JG. (2007). Initiation of RNA decay in Escherichia coli by $5^{\prime}$ pyrophosphate removal. Mol Cell 27: 79-90.

Chisholm SW. (1992). Phytoplankton Size. In: Falkowski PG, Woodhead AD (eds). Primary Productivity and Biogeochemical Cycles in the Sea. Springer, US: Boston, MA, USA pp 213-237.

Deutsch C, Sarmiento JL, Sigman DM, Gruber N, Dunne JP. (2007). Spatial coupling of nitrogen inputs and losses in the ocean. Nature 445: 163-167.

Flombaum P, Gallegos JL, Gordillo RA, Rincón J, Zabala LL, Jiao N et al. (2013). Present and future global distributions of the marine Cyanobacteria Prochlorococcus and Synechococcus. Proc Natl Acad Sci USA 110: 9824-9829.

Gavis J. (1976). Munk and Riley revisited - Nutrient diffusion transport and rates of phytoplankton growth. J Mar Res 34: 161-179.

Gilbert JDJ, Fagan WF. (2011). Contrasting mechanisms of proteomic nitrogen thrift in Prochlorococcus. Mol Ecol 20: 92-104.

Giovannoni SJ, Thrash JC, Temperton B. (2014). Implications of streamlining theory for microbial ecology. ISME J 8: 1553-1565.

Grzymski JJ, Dussaq AM. (2012). The significance of nitrogen cost minimization in proteomes of marine microorganisms. ISME J 6: 71-80.

Grzymski JJ, Marsh AG. (2014). Protein languages differ depending on microorganism lifestyle. Ouzounis CA (ed) PLoS One 9: e96910.

Havaux M, Guedeney G, He Q, Grossman AR. (2003). Elimination of high-light-inducible polypeptides related to eukaryotic chlorophyll a/b-binding proteins results in aberrant photoacclimation in Synechocystis PCC6803. BBA Bioenerg 1557: 21-33.

Hoffmann S, Otto C, Doose G, Tanzer A, Langenberger D, Christ $S$ et al. (2014). A multi-split mapping algorithm for circular RNA, splicing, trans-splicing and fusion detection. Genome Biol 15: R34.

Hoffmann S, Otto C, Kurtz S, Sharma CM, Khaitovich P, Vogel J et al. (2009). Fast mapping of short sequences with mismatches, insertions and deletions using index structures. Searls DB (ed) PLoS Comput Biol 5: e1000502.

Li G-W, Oh E, Weissman JS. (2012). The anti-ShineDalgarno sequence drives translational pausing and codon choice in bacteria. Nature 484: 538-541.

Li H, Handsaker B, Wysoker A, Fennell T, Ruan J, Homer N et al. (2009). The Sequence Alignment/Map format and SAMtools. Bioinformatics 25: 2078-2079.

Lybecker M, Bilusic I, Raghavan R. (2014). Pervasive transcription: detecting functional RNAs in bacteria. Transcription 5: e944039.

Malmstrom RR, Coe A, Kettler GC, Martiny AC, FriasLopez J, Zinser ER et al. (2010). Temporal dynamics of Prochlorococcus ecotypes in the Atlantic and Pacific oceans. ISME J 4: 1252-1264.

McClure R, Balasubramanian D, Sun Y, Bobrovskyy M, Sumby P, Genco CA et al. (2013). Computational analysis of bacterial RNA-Seq data. Nucleic Acids Res 41: e140-e140. 
Mezulis S, Yates CM, Wass MN, Sternberg MJE, Kelley LA. (2015). The Phyre2 web portal for protein modeling, prediction and analysis. Nat Protoc 10: 845-858.

Mitschke J, Georg J, Scholz I, Sharma CM, Dienst D, Bantscheff J et al. (2011). An experimentally anchored map of transcriptional start sites in the model cyanobacterium Synechocystis sp. PCC6803. Proc Natl Acad Sci USA 108: 2124-2129.

Moll I, Grill S, Gualerzi CO, Bläsi U. (2002). Leaderless mRNAs in bacteria: surprises in ribosomal recruitment and translational control. Mol Microbiol 43: 239-246.

Moore CM, Mills MM, Arrigo KR, Berman-Frank I, Bopp L, Boyd PW et al. (2013). Processes and patterns of oceanic nutrient limitation. Nat Geosci 6: 701-710.

Moore LR, Coe A, Zinser ER, Saito MA, Sullivan MB, Lindell D et al. (2007). Culturing the marine cyanobacterium Prochlorococcus. Limnol Oceanogr Met 5: 353-362.

Munk WH, Riley GA. (1952). Absorption of nutrients by aquatic plants. J Mar Res 11: 215-240.

Mutsuda M, Sugiura M. (2006). Translation initiation of cyanobacterial rbcS mRNAs requires the $38-\mathrm{kDa}$ ribosomal protein S1 but not the Shine-Dalgarno sequence: development of a cyanobacterial in vitro translation system. J Biol Chem 281: 38314-38321.

Ohashi Y, Shi W, Takatani N, Aichi M, Maeda S-I, Watanabe S et al. (2011). Regulation of nitrate assimilation in cyanobacteria. J Exp Bot 62: 1411-1424.

Olson RJ, Vaulot D, Chisholm SW. (1985). Marine phytoplankton distributions measured using shipboard flow cytometry. DeepSea Res 32: 1273-1280.

Parkhill JP, Maillet G, Cullen JJ. (2001). Fluorescence-based maximal quantum yield for PSII as a diagnostic of nutrient stress. J Phycol 37: 517-529.

Robinson JT, Thorvaldsdottir H, Winckler W, Guttman M, Lander ES, Getz G et al. (2011). Integrative genomics viewer. Nat Biotech 29: 24-26.

Sauer J, Görl M, Forchhammer K. (1999). Nitrogen starvation in Synechococcus PCC 7942: involvement of glutamine synthetase and NtcA in phycobiliprotein degradation and survival. Arch Microbiol 172: 247-255.

Schoenberg DR. (2007). The end defines the means in bacterial mRNA decay. Nat Chem Biol 3: 535-536.

Shao W, Price MN, Deutschbauer AM, Romine MF, Arkin AP. (2014). Conservation of transcription start sites within genes across a bacterial genus. MBio 5: e01398-14e01398-14.

Sharma CM, Hoffmann S, Darfeuille F, Reignier J, Findeiß S, Sittka A et al. (2010). The primary transcriptome of the major human pathogen Helicobacter pylori. Nature 464: 250-255.

Sherman MY, Qian S-B. (2013). Less is more: improving proteostasis by translation slow down. Trends Biochem Sci 38: 585-591.

Sowell SM, Wilhelm LJ, Norbeck AD, Lipton MS, Nicora CD, Barofsky DF et al. (2008). Transport functions dominate the SAR11 metaproteome at low-nutrient extremes in the Sargasso Sea. ISME J 3: 93-105.

Steglich C, Futschik ME, Lindell D, Voss B, Chisholm SW, Hess WR. (2008). The challenge of regulation in a minimal photoautotroph: non-coding RNAs in Prochlorococcus. PLoS Genet 4: e1000173.

Steglich C, Lindell D, Futschik M, Rector T, Steen R, Chisholm SW. (2010). Short RNA half-lives in the slow-growing marine cyanobacterium Prochlorococcus. Genome Biol 11: R54.

$\mathrm{Su}$ Z. (2005). Comparative genomics analysis of NtcA regulons in cyanobacteria: regulation of nitrogen assimilation and its coupling to photosynthesis. Nucleic Acids Res 33: 5156-5171.

Thomason MK, Bischler T, Eisenbart SK, Förstner KU, Zhang A, Herbig A et al. (2015). Global transcriptional start site mapping using differential RNA sequencing reveals novel antisense RNAs in Escherichia coli. J Bacteriol 197: 18-28.

Thompson AW, Huang K, Saito MA, Chisholm SW. (2011). Transcriptome response of high- and low-light-adapted Prochlorococcus strains to changing iron availability. ISME J 5: 1580-1594.

Thorvaldsdóttir H, Robinson JT, Mesirov JP. (2013). Integrative Genomics Viewer (IGV): high-performance genomics data visualization and exploration. Brief Bioinform 14: 178-192.

Tjaden B. (2015). De novo assembly of bacterial transcriptomes from RNA-seq data. Genome Biol 16: 1.

Tolonen AC, Aach J, Lindell D, Johnson ZI, Rector T, Steen R et al. (2006). Global gene expression of Prochlorococcus ecotypes in response to changes in nitrogen availability. Mol Syst Biol 2: 53.

Tyrrell T. (1999). The relative influences of nitrogen and phosphorus on oceanic primary production. Nature 400: $525-531$.

Van Mooy BAS, Rocap G, Fredricks HF, Evans CT, Devol AH. (2006). Sulfolipids dramatically decrease phosphorus demand by picocyanobacteria in oligotrophic marine environments. Proc Natl Acad Sci USA 103: 8607-8612.

Voigt K, Sharma CM, Mitschke J, Lambrecht SJ, Voß B, Hess WR et al. (2014). Comparative transcriptomics of two environmentally relevant cyanobacteria reveals unexpected transcriptome diversity. ISME J 8: 2056-2068.

Wickham H. (2009). ggplot2: elegant graphics for data analysis. Springer: New York.

Zinser ER, Coe A, Johnson ZI, Martiny AC, Fuller NJ, Scanlan DJ et al. (2006). Prochlorococcus ecotype abundances in the North Atlantic Ocean as revealed by an improved quantitative PCR method. Appl Environ Microbiol 72: 723-732.

Zinser ER, Johnson ZI, Coe A, Karaca E, Veneziano D, Chisholm SW. (2007). Influence of light and temperature on Prochlorococcus ecotype distributions in the Atlantic Ocean. Limnol and Oceanogr 52: 2205-2220.

This work is licensed under a Creative Commons Attribution 4.0 International License. The images or other third party material in this article are included in the article's Creative Commons license, unless indicated otherwise in the credit line; if the material is not included under the Creative Commons license, users will need to obtain permission from the license holder to reproduce the material. To view a copy of this license, visit http:// creativecommons.org/licenses/by/4.0/

(C) The Author(s) 2017

Supplementary Information accompanies this paper on The ISME Journal website (http://www.nature.com/ismej) 